\title{
Testin (TES) as a candidate tumour suppressor and prognostic marker in human astrocytoma
}

\author{
GIEDRIUS STEPONAITIS, ARUNAS KAZLAUSKAS, DAINA SKIRIUTE, INDRE VALIULYTE, \\ KESTUTIS SKAUMINAS, ARIMANTAS TAMASAUSKAS and PAULINA VAITKIENE
}

Laboratory of Neuro-Oncology and Genetics, Neuroscience Institute, Lithuanian University of Health Sciences, LT-50009 Kaunas, Lithuania

Received April 1, 2016; Accepted July 22, 2016

DOI: $10.3892 / \mathrm{ol} .2016 .5077$

\begin{abstract}
Astrocytomas are one of the most common brain tumours; however, the current methods used to characterize these tumours are inadequate. The establishment of molecular markers may identify variables required to improve tumour characterization and subtyping, and may aid to specify targets for improved treatment with essential prognostic value for patient survival. One such candidate is testin (TES), which was reported to have prognostic value for glioblastoma patients. However, the role of TES protein in gliomagenesis is currently unknown. In the present study, the methylation status of the TES promoter was investigated in post-operative astrocytoma tumours of different malignancy grade, and its association with the survival of astrocytoma patients was evaluated. In addition, the expression of TES protein was investigated in the same set of astrocytoma tumours tissue, and the association of protein expression with glioma patients survival was evaluated. The methylation status of TES was assessed by methylation-specific polymerase chain reaction in 138 different grade astrocytoma samples. Western blot analysis was used to characterize the expression pattern of TES in 86 different grade astrocytoma specimens: 13 of pathological grade I, 31 of pathological grade II, 17 of pathological grade III and 25 of pathological grade IV (glioblastoma). Statistical analyses were conducted to investigate the association between tumour molecular pattern, patient clinical variables and overall survival. The methylation analysis of the TES promoter exhibited a distinct profile between astrocytomas of different malignancy grade $(\mathrm{P}<0.001)$. Furthermore, gene promoter methylation was significantly associated with patients' age, survival and pathological grade
\end{abstract}

Correspondence to: Dr Paulina Vaitkiene, Laboratory of Neuro-Oncology and Genetics, Neuroscience Institute, Lithuanian University of Health Sciences, Eiveniu Street 4, LT-50009 Kaunas, Lithuania

E-mail: paulina.vaitkiene@lsmuni.lt

Key words: testin, testin LIM domain protein, testis-derived transcript, astrocytoma, glioma, glioblastoma, DNA methylation, expression, survival
$(\mathrm{P}<0.001)$. The protein expression level of TES was significantly lower in glioblastoma (grade IV astrocytoma) than in lower grade (II-III) astrocytoma tissue $(\mathrm{P}=0.028$ and $\mathrm{P}=0.04$, respectively). Additionally, short overall survival of patients was markedly associated with low TES protein expression $(\mathrm{P}=0.007)$. However, no association between TES methylation and TES protein expression was noticed. The present study demonstrated that decreased expression of TES may be important in tumour progression and prognosis in human astrocytomas. TES may be a useful marker for predicting the clinical outcome of astrocytoma patients.

\section{Introduction}

Astrocytomas are one of the most common brain tumours, and are classified as grades I to IV based on histology and clinical criteria (1). Although low-grade astrocytoma patients have better survival compared with high-grade astrocytoma patients (World Health Organization grade III or IV), the majority of tumours eventually progress to high-grade astrocytoma and cause patient mortality (2). Various clinical characteristics fail to explain a variation in disease progression in histologically diagnosed same-grade astrocytomas (3). It has been proposed that historically used clinical variables are not sufficient prognostic or predictive indicators, as compared with the information provided by molecular markers of tumours (2). Furthermore, the discovery of molecular markers may identify suitable treatments for sensitive patients and novel targets for improved treatment in the future (2).

Gliomagenesis is a multistep process resulting from the accumulation of genetic and epigenetic changes. DNA methylation is one of the major epigenetic modifications, and leads to gene silencing (4). The frequency of hypermethylation of $\mathrm{CpG}$ dinucleotides varies significantly between different malignancy grades of gliomas (5). Previous data in breast cancer suggest the possibility that down-regulation of testin (TES) is associated with alterations in cell adhesion and motility, and therefore may lead to the development of tumours with an aggressive phenotype (6).

The TES gene, also known as testin LIM domain protein, is located on chromosome 7q31.2 (7). The protein encoded by the TES gene is a negative regulator of cell growth and may 
act as a tumour suppressor. This protein may also play a role in cell adhesion, cell spreading and reorganization of the actin cytoskeleton (8). The loss of TES expression occurs frequently in various cancers $(7,9)$. Missense mutations are scarce, and homozygous deletions have not been observed, which is consistent with the fact that $\mathrm{CpG}$ promoter hypermethylation is a mechanism of TES inactivation (9). TES methylation has been reported in primary tumours, including glioblastomas (10). Promoter methylation is closely associated with the loss of TES expression in glioblastoma cell lines (10). The role of TES promoter methylation and TES protein expression in low-grade astrocytomas has not been studied thus far. Additionally, there are limited data on TES molecular alterations in high-grade astrocytomas and on the influence of these alterations in patient clinical outcome. The present study investigated whether TES gene promoter methylation and TES expression are associated with glioma malignancy and participate in gliomagenesis.

\section{Materials and methods}

Patients and tissue samples. In total, 138 post-operative glioma tumour samples of different malignancy grade were collected in the Neurosurgery Clinic Hospital of the Lithuanian University of Health Sciences (Kaunas, Lithuania) between March 2003 and January 2013. Informed consent was obtained from patients. The study was performed in accordance with the principles of the Declaration of Helsinki, and was approved by the Ethics Committee for Biomedical Research of the Lithuanian University of Health Sciences.

In total, 138 patients diagnosed with different malignancy grade of astrocytoma tumour were involved in the analysis: Grade I, 14 samples; grade II, 46 samples; grade III, 29 samples; and grade IV (glioblastoma), 49 samples. Diagnoses were established by experienced pathologists according to the WHO classification (11). Following resection, glioma samples were immediately stored in liquid nitrogen (snap frozen) until DNA extraction and protein lysate preparation. The following clinical data were collected for each patient: Age at the time of the operation, gender, time of the last follow-up and patient status. For the prognostic analysis, patient survival was calculated from the date of the operation until mortality, or until the date of termination of the study. None of the patients had received chemotherapy or radiation prior to surgery.

DNA isolation and bisulfate modification. Tumour DNA was extracted from 50-150 mg of snap-frozen tissue. Cryogenic mechanical tissue disruption was used for homogenization of tissue. DNA was purified using the salting-out method, as described by Miller et al (12) with one modification: $50 \mu \mathrm{l}$ chloroform was used for $850 \mu \mathrm{l}$ of the total lysate following the $6 \mathrm{M} \mathrm{NaCl}$ step. The methylation status of the TES gene promoter was determined upon bisulfite treatment of the DNA. A total of $400 \mathrm{ng}$ DNA was used for bisulfite modification. DNA modification was performed using the EZ DNA Methylation kit (Zymo Research Corporation, Irvine, CA, USA), and all procedures were conducted according to the manufacturer's protocol. Bisulfite-treated DNA was eluted in $40 \mu \mathrm{l}$ distilled water, and stored at $-80^{\circ} \mathrm{C}$ until subjected to methylation-specific polymerase chain reaction (MS-PCR).
$M S-P C R$. The methylation status of the TES promoter region was determined by MS-PCR. Primers distinguishing unmethylated from methylated alleles were previously published by Ma et al (13), and their sequences were as follows: Methylated forward: 5'-TATTGAGTTTGTTTAGTA GGGCGTC-3' and reverse: 5'-AATAACAACCGAACAACT CCG-3'; and unmethylated forward: 5'-TGAGTTTGTTTA GTAGGGTGTTG-3' and reverse: 5'-ATAACAACCAAA CAACTCCAA-3'. Each PCR reaction incorporated $20 \mathrm{ng}$ of sodium bisulphite-modified DNA. MS-PCR was performed in a total volume of $15 \mu \mathrm{l}$, using $7.5 \mu \mathrm{l}$ Maxima Hot Start Green PCR Master Mix (2X) with Hot Start Taq DNA Polymerase (Thermo Fisher Scientific, Inc., Waltham, MA, USA) and $10 \mathrm{pmol}$ of each primer (Metabion International AG, Steinkirchen, Germany). The cycling conditions were as follows: Initial denaturation at $95^{\circ} \mathrm{C}$ for $5 \mathrm{~min}$, followed by 36 cycles of $94^{\circ} \mathrm{C}$ for $15 \mathrm{sec}, 62^{\circ} \mathrm{C}$ for $30 \mathrm{sec}$ and $72^{\circ} \mathrm{C}$ for $15 \mathrm{sec}$, and a final step at $72^{\circ} \mathrm{C}$ for $5 \mathrm{~min}$. For each set of MS-PCR, human blood lymphocyte DNA treated with bisulfite (Zymo Research Corporation) served as an unmethylated DNA control, and as a positive methylation control, Bisulfite-converted Universal Methylated Human DNA Standard (Zymo Research Corporation) was used. Human Matched DNA (Zymo Research Corporation) served as a normal brain tissue control. A water blank control was also included. The PCR products were analysed on $2 \%$ agarose gels with ethidium bromide, and visualized under ultraviolet illumination. PCR analyses were repeated twice for each sample.

Whole-tissue extract preparation and western blot analysis. Whole-tissue extracts of cryogenically homogenized tumour samples were routinely prepared by resuspending the disaggregated samples (100-200 mg) in radioimmunoprecipitation assay lysis buffer [50 mM Tris- $\mathrm{HCl}$ (pH 7.5), $150 \mathrm{mM} \mathrm{NaCl}$, 1\% IGEPAL ${ }^{\circledR}$ CA-630 (Cat. No. I3021; Sigma-Aldrich; Merck Millipore, Darmstadt, Germany), $0.5 \%$ sodium deoxycholate and $0.1 \%$ sodium dodecyl sulfate (SDS)] supplemented with a protease inhibitor cocktail (Cat. No. S8820; Sigma-Aldrich; Merck Millipore), and next homogenized using an ultrasonic sonicator (500-Watt Ultrasonic Processor; Cole-Parmer Instrument Co. Ltd., London, UK). Subsequently, the extracts were cleared by centrifugation for $30 \mathrm{~min}$ at $13,000 \mathrm{x} \mathrm{g}$ at $4^{\circ} \mathrm{C}$. In total, $80 \mu \mathrm{g}$ of the total extract of protein were fractionated by $7.5 \%$ SDS-polyacrylamide gel electrophoresis and transferred to nitrocellulose membranes. Each membrane incorporated a full set of analysed astrocytoma malignancies of grades I-IV. The immobilized proteins were incubated for $4 \mathrm{~h}$ at room temperature under rotation with a primary rabbit antibody against TES (dilution 1:500; Cat. No. 10258-1-AP; ProteinTech Group, Inc., Chicago, IL, USA) in blocking solution [5\% non-fat milk in phosphate-buffered saline (PBS)]. Upon extensive washing in PBS supplemented with $0.5 \%$ Tween-20 buffer, membranes were incubated with a horseradish peroxidase (HRP)-conjugated anti-rabbit secondary antibody (dilution 1:2,000; Cat. No. 656120; Thermo Fisher Scientific, Inc.) for $1 \mathrm{~h}$ at room temperature under rotation. For detection of $\beta$-actin on the same membranes, the the TES antibody complexes were first cleared from the membranes by washing in a mild striping 
Table I. Association between TES promoter methylation and clinicopathological features in human astrocytoma tissue.

\begin{tabular}{lccc}
\hline & \multicolumn{2}{c}{ TES gene promoter status } & P-value \\
\cline { 2 - 3 } Variables & Methylated, $\mathrm{n}(\%)$ & Unmethylated, $\mathrm{n}(\%)$ & $<0.001$ \\
\hline Total patients & $79(57.25)$ & $59(42.75)$ & \\
Age (years) & $39(44.80)$ & $48(55.20)$ & 0.730 \\
$\leq 55$ & $40(78.40)$ & $11(21.60)$ & $<0.001$ \\
$>55$ & & & \\
Gender & $34(54.84)$ & $28(45.16)$ & $<0.001$ \\
Male & $45(59.21)$ & $31(40.79)$ & \\
Female & & $13(22.81)$ & \\
Survival (months) & $44(77.19)$ & $46(56.79)$ & \\
$\leq 24$ & $35(43.21)$ & $14(100.00)$ & \\
$>24$ & $0(0.00)$ & $25(54.35)$ & \\
Pathological grade & $21(45.65)$ & $10(34.48)$ & \\
I & $19(65.52)$ & $(20.41)$ & \\
II & $39(79.59)$ & & \\
III & & & \\
IV & & & \\
\hline
\end{tabular}

TES promoter methylation status data were divided into two categories: Methylated and unmethylated. The differences between age, gender and pathological grade in each category were analysed by the $\chi^{2}$ method. TES, testin.

buffer (25 mM glycine and $2 \%$ SDS, pH 2.0), and then the membranes were re-probed with a primary monoclonal mouse antibody against $\beta$-actin (dilution 1:2,000; Cat. No. ABIN559692; Antibodies-online Inc., Atlanta, GA, USA) for $1 \mathrm{~h}$ at room temperature under rotation, followed by incubation with an HRP-conjugated anti-mouse secondary antibody (dilution 1:2,000; Cat. No. 626520; Thermo Fisher Scientific, Inc.) for $1 \mathrm{~h}$ at room temperature. Immunocomplexes were visualized using liquid 3,3',5,5'-tetramethylbenzidine substrate (Cat. No. T0565-100ML; Sigma-Aldrich; Merck Millipore) and documented using an ordinary scanner.

The values of TES and $\beta$-actin signals were calculated using the image analysis program ImageJ version 1.47 (National Institutes of Health, Bethesda, USA). It should be noted that when calculating TES signals, all bands ( 48 kDa full-length TES, as described in the literature (8), and possible degradation products of the protein) were included in the analysis.

Statistical analysis. SPSS version 19 software (IBM SPSS, Armonk, NY, USA) was used for statistical analysis. Associations between gene methylation data and clinical features of glioma patients were analysed by the $\chi^{2}$ test. To estimate survival functions, the Kaplan-Meier method was used. For comparing survival time distributions between groups, the log-rank test was applied. For determination of the independence of prognostic factors, the Cox regression analysis was used. For western blot assays, differences across groups were analysed using the Kruskal-Wallis test (comparison of $>2$ groups). To evaluate expression differences across different gene methylation groups, the Mann-Whitney test was used (comparison of two groups). For the analysis of patients prognosis, survival was calculated from the date of operation until mortality or until the date of the last follow-up. $\mathrm{P}<0.05$ was considered to indicate a statistically significant difference.

\section{Results}

TES promoter is hypermethylated in glioblastoma tumours. The methylation status of the TES promoter in glioblastoma tumour samples was detected by MS-PCR assay. The methylation status of the TES promoter was evaluated in 138 astrocytoma tumours: Grade I, 14 samples; grade II, 46 samples; grade III, 29 samples; and grade IV (glioblastoma), 49 samples. Promoter hypermethylation was detected in $57.25 \%$ (79/138) of all analysed astrocytoma tumours, but not in normal brain tissue. The results revealed that the TES gene methylation frequency increases with the degree of malignancy of the tumour (Fig. 1A).

Methylated TES gene promoter was not detected in grade I astrocytoma $[0.00 \%(0 / 14)]$, while almost half of the grade II astrocytoma samples were methylated [45.65\% (21/46)]. Grade III astrocytoma TES promoter methylation was detected in $65.52 \%(19 / 29)$ of samples, and the highest methylation degree of the TES gene was observed in glioblastoma samples [79.59\% (39/49)]. It should be mentioned that unmethylated promoter of the TES gene was detected in the normal brain control.

The TES gene methylation status differed significantly between astrocytomas of different malignancy grades $(\mathrm{P}<0.001)$. The present study revealed a significant association between patients' clinical data and survival, and TES gene promoter methylation $(\mathrm{P}<0.05)$ (Table I).

The results revealed that methylated TES promoter was more common (78.4\%) in patients older than 55 years as compared with younger patients (55.2\%). In addition, 
Table II. Association between TES expression in human glioma tissues and different clinicopathological features.

\begin{tabular}{|c|c|c|c|c|c|}
\hline \multirow[b]{2}{*}{ Variable } & \multirow[b]{2}{*}{ Cases, $\mathrm{n}$} & \multicolumn{3}{|c|}{ TES expression } & \multirow[b]{2}{*}{ P-value } \\
\hline & & Low, n (\%) & Medium, n (\%) & High, n (\%) & \\
\hline Total patients & 86 & $22(25.6)$ & $42(48.8)$ & $22(25.6)$ & \\
\hline Age (years) & & & & & 0.228 \\
\hline$\leq 55$ & 58 & $13(22.4)$ & 27 (46.6) & $18(31.0)$ & \\
\hline$>55$ & 28 & $9(32.1)$ & $15(53.6)$ & $4(14.3)$ & \\
\hline Gender & & & & & 0.472 \\
\hline Male & 40 & $8(20.0)$ & $22(55.0)$ & $10(25.0)$ & \\
\hline Female & 46 & $14(30.4)$ & $20(43.5)$ & $12(26.1)$ & \\
\hline Pathological grade & & & & & 0.012 \\
\hline I & 13 & $3(23.1)$ & $7(54.8)$ & $3(23.1)$ & \\
\hline II & 31 & $5(16.1)$ & $17(54.8)$ & $9(29.1)$ & \\
\hline III & 17 & $3(17.6)$ & $7(41.2)$ & $7(41.2)$ & \\
\hline IV & 25 & $11(44.0)$ & $11(44.0)$ & $3(12.0)$ & \\
\hline
\end{tabular}

TES protein level data were divided into three categories: Low, medium and high. In each category, associations of TES expression with age, gender and pathological grade were defined. P-value was estimated by the $\chi^{2}$ method. TES, testin.

A

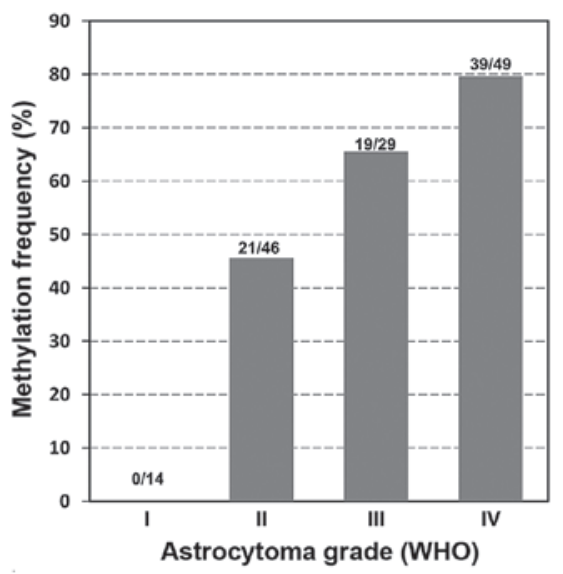

B

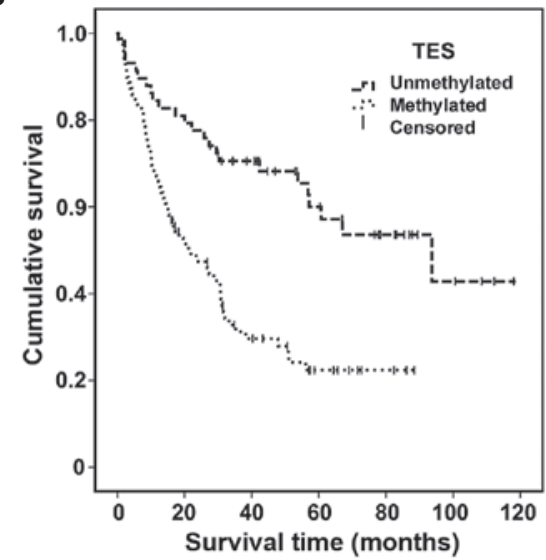

Figure 1.TES promoter methylation analysis in astrocytomas. (A) Frequency of methylated TES gene promoter in different malignancy grade astrocytomas. (B) Kaplan-Meier estimation of overall survival of astrocytoma patients (all grades) according to the methylation status of the TES gene promoter (log-rank test, $\chi^{2}=17.864$, degrees of freedom $\left.=1, \mathrm{P}<0.001\right)$. TES, testin; WHO, World Health Organization.

methylation of TES was significantly associated with patient survival. Patients who survived $<24$ months after resection tended to have a methylated TES allele $(\mathrm{P}=0.001)$.

However, there was no significant association between TES methylation status and patient gender.

The use of the methylation of the TES gene promoter as a prognostic value of overall survival was evaluated using Kaplan-Meier analysis (Fig. 1B). Promoter methylation of TES was noticed to be closely associated with shorter survival (long-rank test, $\mathrm{P}<0.001$ ).

TES protein expression in glioma. Hypermethylation typically occurs at $\mathrm{CpG}$ islands in the promoter region of genes, and is associated with gene silencing (4). To determine if TES protein expression was associated with tumour histopathological grading and TES gene promoter methylation, the aforementioned set of 86 human astrocytoma samples (13 of grade I, 31 of grade II, 17 of grade III and 25 of grade IV) was used for TES western blot analysis.

The protein expression levels of TES in glioblastoma (grade IV) were significantly lower than those in lower grade (II-III) glioma tissues $(\mathrm{P}<0.05)$ (Fig. 2A and $\mathrm{B})$. To determine the significance of decreased TES expression in glioblastoma, the association between TES expression and clinicopathological features of astrocytoma patients was assessed. For this purpose, the TES protein expression values obtained by western blotting were ranked into three categories irrespective of tumour grading: Values that were lower than or equal to the 25th percentile were ranked as 'low' TES protein expression; values ranging from the 25 th to the 75 th percentiles were considered as 'medium' TES protein expression; and values that were higher than or equal to the 75 th percentile 
A

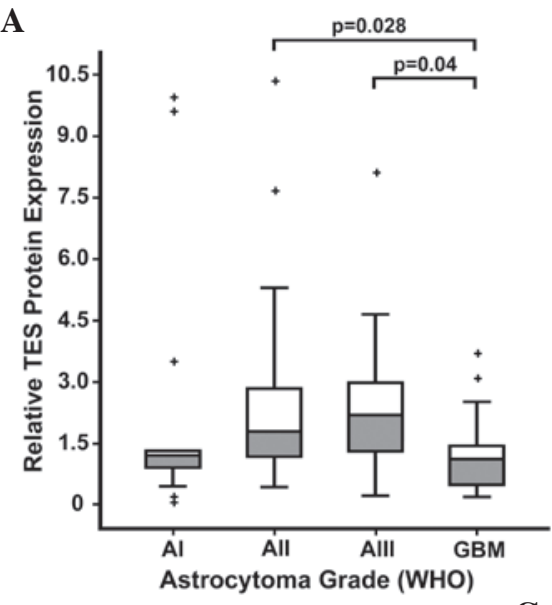

B

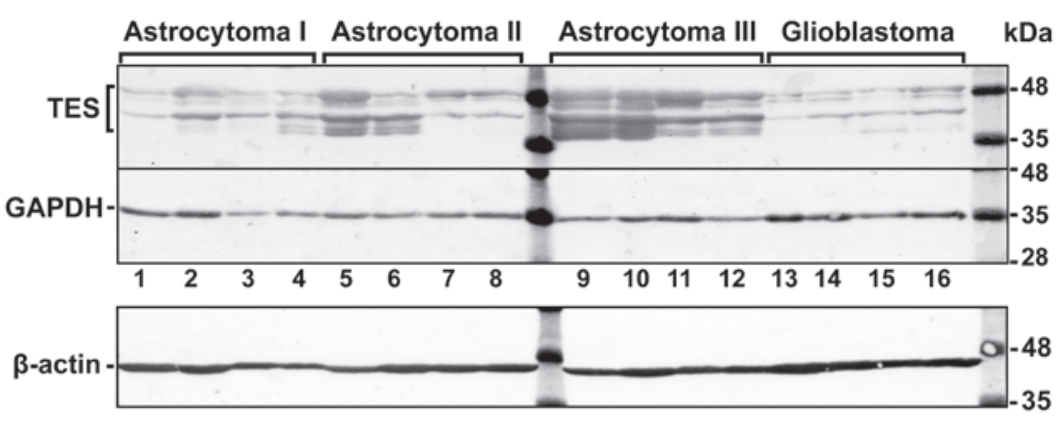

C

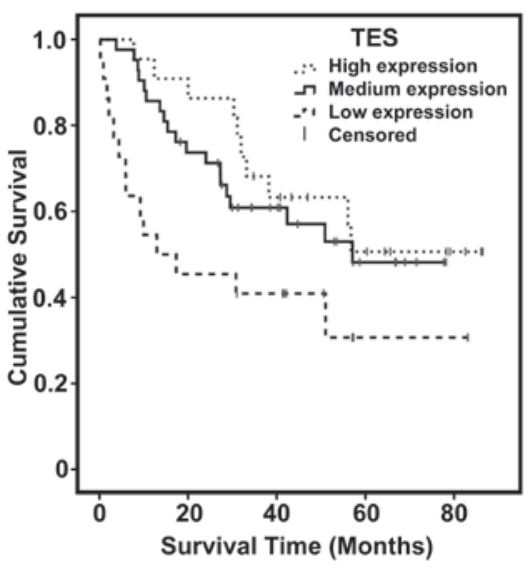

Figure 2. Analysis of TES protein expression in astrocytomas. (A) Relative TES protein expression in different malignancy grade astrocytomas. Box plots of relative expression measurements of TES obtained by western blot analysis of astrocytoma samples. Pathological grades I, II, III and IV are indicated as AI, AII, AIII and GBM, respectively. The line inside each box represents the median; the lower and upper edges of the boxes represent the 25th (1st quartile) and 75th (3rd quartile) percentiles, respectively; and the upper and lower edges of the boxes represent the Tukey's whiskers. The plus (+) symbols represent the outliers (values greater than 1.5 interquartile ranges below the 1st or above the 3rd quartile). (B) Representative western blot results of TES protein expression in astrocytoma. The highest molecular weight band represents the TES protein $(\sim 48 \mathrm{kDa})$, while the other bands observed below may be possible degradation products of TES. In addition, the $\sim 42 \mathrm{kDa}$ band that is steadily visible in all samples could be a plausible isoform of TES. GADPH in this example was re-probed on the same membrane, while $\beta$-actin was detected on other membrane with the same set and arrangement of samples. (C) Kaplan-Meier survival curves of astrocytoma patients stratified by TES protein expression groups (high, medium and low) (log-rank test, $\chi^{2}=6.285$, degrees of freedom $=2, \mathrm{P}=0.043$ ). TES, testin; GBM, glioblastoma; WHO, World Health Organization; GAPDH, glyceraldehyde 3-phosphate dehydrogenase.

were ranked as 'high' TES protein expression. According to the western blot results, low expression levels of TES were determined for $22(25.6 \%)$ cases, medium expression levels were determined for $42(48.8 \%)$ cases and high expression levels were determined for $22(25.6 \%)$ cases of the total 86 glioma patients. As indicated in Table II, according to the Kruskal-Wallis test, TES expression differed significantly between different astrocytoma grades $(\mathrm{P}=0.012)$. Pairwise comparison to assess the differences for each group revealed that grade II and grade III tumours tended to have significantly higher TES protein expression than glioblastomas $(\mathrm{P}=0.028$ and $\mathrm{P}=0.040$, respectively) (Fig. $2 \mathrm{~A}$ ). This suggests that decreased TES expression could be positively associated with glioma progression. The TES protein levels did not correlate with patients' age or gender (both $\mathrm{P}>0.05$ ) (Table II).

Next, Kaplan-Meier analysis was performed using the log-rank test to determine the association between TES expression and clinical outcome of glioma patients (Fig. 2C). The analysis revealed that patients with low TES protein expression level had significantly lower rate of overall survival when compared with medium and high TES expression patients $\left(\mathrm{P}=0.014\right.$; degrees of freedom $\left.=2 ; \chi^{2}=8.2\right)$ (Fig. $\left.2 \mathrm{~B}\right)$. The survival analysis data suggested an association between TES expression and astrocytoma grade, and indicated that decreased level of TES could be associated with tumour progression.

This hypothetical association between TES promoter methylation and TES protein expression prompted us to examine whether TES protein level differed in TES promoter methylation groups of various grades. TES protein expression data were divided into two groups according to gene promoter methylation status: Methylated TES promoter and unmethylated TES promoter in each WHO grade. No significant association between TES protein expression and TES promoter methylation was observed in any of the WHO groups analysed, which was most likely due to the small number of cases (Fig. 3). Furthermore, in addition to methylation, TES down-regulation could be caused by other molecular mechanisms, including mutation, loss of heterozygosity and microRNA regulation, which may affect the correlation between gene expression and methylation. 


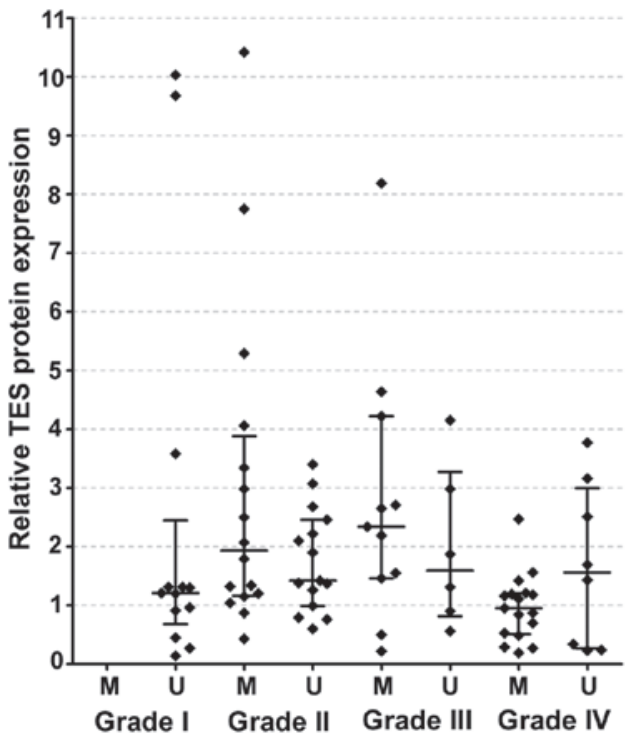

Figure 3. Distributions of relative TES protein expression in different astrocytoma grade and methylation groups. The middle line in each group represents the median, while and the upper and lower lines represent the upper and lower limits of the interquartile range, respectively. No significant association between TES promoter methylation status and TES protein expression was observed. TES, testin; M, methylated; U, unmethylated.

Cox regression analysis was performed to assess the prognostic independence of the measured variables. Molecular variables such as TES gene promoter methylation and TES protein expression, and clinical data such as astrocytoma pathological grade, patients' age and gender, were selected to be tested. Univariate Cox analysis revealed that TES protein expression $[\mathrm{P}=0.020$; hazard ratio (HR), 0.75], gene promoter methylation $(\mathrm{P}=0.005$; HR, 0.5), tumour pathological grade $(\mathrm{P}<0.001)$ and patients' age $(\mathrm{P}<0.001)$ but not gender $(\mathrm{P}=0.644)$ had a significant association as independent variables with the patients' overall survival. All the variables that had a strong impact on survival $(\mathrm{P}<0.05)$ were then assessed jointly in a multivariate Cox analysis. However, the results from the multivariate analysis did not confirm that TES methylation or TES protein expression were independent prognostic factors ( $\mathrm{P}=0.366$ and $\mathrm{P}=0.219$, respectively), although it confirmed the prognostic independence character of tumour pathological grade $(\mathrm{P}=0.008)$ and patients' age $(\mathrm{P}=0.001)$. Therefore, future studies with larger sample sizes should be conducted to confirm this tendency.

\section{Discussion}

Epigenetic changes, including aberrant DNA methylation, are important in the pathogenesis of glial tumours $(4,14)$. Thus, the search for novel methylation biomarkers or the validation of already identified methylation biomarkers, which may facilitate glioma diagnosis, prognosis or treatment decisions, remains a current issue (14). In the present study, the methylation status of the TES gene, which has the potential to be used in routine clinical setting as a biomarker for astrocytomas of different malignancy grade, was analysed. Previous data have suggested the possibility that down-regulation of TES is associated with alterations in cell adhesion and motility, and therefore, may lead to the development of tumours with an aggressive phenotype (6). The TES gene was noticed to be highly methylated in glioblastomas $(5,10,15)$, and the tumour-associated methylation of TES was confirmed in cultured glioblastomas and glioblastoma cell lines (10). These results indicate that TES methylation may be involved in the loss of inter-networks in cells, which allows them to migrate, leading to tumour infiltration of the brain. Gunduz et al reported an association between TES down-regulation and poor patients' outcome in head and neck squamous cell carcinomas (16). The down-regulation of $T E S$ was demonstrated to be correlated with tumour differentiation and prognosis in gastric cancer (13), and TES was densely methylated in acute lymphoblastic leukemia (17). There is limited information about TES methylation in astrocytomas. It has been reported that $T E S$ appears to be frequently methylated in glioblastoma (58-69\%) $(5,10,15)$.

In agreement with previous studies $(5,10,15)$, the present study demonstrated a similar TES methylation frequency (79.6\%) in glioblastoma. In addition, the current study revealed that TES gene promoter was unmethlyated in grade I astrocytomas, whereas substantial TES gene promoter methylation was identified in grade II $(45 \%)$ and grade III $(65 \%)$ astrocytoma samples. Aligning the methylation frequency of low-grade astrocytomas with glioblastoma, it was noticed that $T E S$ gene promoter methylation in astrocytomas occurs in the late stages of tumour development. Due to TES gene promoter methylation, gene expression may be lost, which may be associated with the increased tumour invasiveness of gliomas.

The frequency of promoter methylation of the TES gene based on the current MS-PCR results correlated with tumour grade according to the WHO classification criteria, and also with patients' age. Aggressive and invasive astrocytomas (WHO grades IV and III) exhibited a higher frequency of methylated TES promoter compared with low-grade tumours.

The results revealed that the overall survival of patients with TES hypermethylation is significantly shorter than those with hypomethylation. These observations suggest that analyses based on the methylation status of the TES gene promoter could potentially be useful as diagnostic or prognostic tools in the case of tumours for which the histopathological examination is uncertain. However, it remains to be determined whether the increasing rate of TES methylation is associated with the aging process or with the tumour characteristics, since it is usually difficult to separate these two processes.

A previous study by Bai et al, where 37 different glioblastoma specimens were analysed, identified reduced TES immunostaining (compared with normal human brain tissue) in a subpopulation of glioblastoma cells (18). The role of TES protein expression in low-grade astrocytomas according the data obtained in the present study remains to be investigated. To the best of our knowledge, the present study examined for the first time TES expression at protein levels in astrocytoma tissues of different malignancy grade. Using western blotting, a decreased protein level of TES in glioblastomas was detected, compared with lower grade astrocytomas, thus indicating that aberrant expression of TES may be associated with malignant progression of astrocytomas. In addition, TES down-regulation was distinctly associated with poor prognosis in astrocytoma patients, particularly in those with high WHO grade. The present results suggest that down-regulation of 
TES predicts a worse outcome of glioblastoma patients, and that promoter hypermethylation could be one of the reasons for TES down-regulation, although mutation and loss of heterozygosity cannot be excluded. Thus, these results indicate that promoter hypermethylation may present a potential diagnostic tool in the future.

\section{Acknowledgements}

The present study was funded by a grant (No. LIG-11/2012) from the Research Council of Lithuania (Vilnius, Lithuania).

\section{References}

1. Kostoro J, Chang SJ, Clark Lai YC, Wu CC, Chai CY and Kwan AL: Overexpression of vascular adhesion protein-1 is associated with poor prognosis of astrocytomas. APMIS 124: 462-468, 2016.

2. Claus EB, Walsh KM, Wiencke JK, Molinaro AM, Wiemels JL, Schildkraut JM, Bondy ML, Berger M, Jenkins R and Wrensch M: Survival and low-grade glioma: The emergence of genetic information. Neurosurg Focus 38: E6, 2015.

3. Verma V and Mehta MP: Clinical ramifications of "genomic staging" of low-grade gliomas. J Neurooncol: Jul 11, 2016 (Epub ahead of print).

4. Rasime K: Epigenetics of glioblastoma multiforme. J Clinic Res Bioeth 6: 6, 2015.

5. Laffaire J, Everhard S, Idbaih A, Crinière E, Marie Y, de Reyniès A, Schiappa R, Mokhtari K, Hoang-Xuan K, Sanson M, et al: Methylation profiling identifies 2 groups of gliomas according to their tumorigenesis. Neuro Oncol 13 84-98, 2011.

6. Sarti M, Pinton S, Limoni C, Carbone GM, Pagani O, Cavalli F and Catapano CV: Differential expression of testin and survivin in breast cancer subtypes. Oncol Rep 30: 824-832, 2013.

7. Tatarelli C, Linnenbach A, Mimori K and Croce CM: Characterization of the human TESTIN gene localized in the FRA7 G region at 7q31.2. Genomics 68: 1-12, 2000.

8. The Human Protein Atlas: v15.proteinatlas.org. Testis derived transcript (3 LIM domains). http://www.proteinatlas. org/ENSG00000135269-TES/gene. Accessed Jun 10, 2016.
9. Tobias ES, Hurlstone AF, MacKenzie E, McFarlane R and Black DM: The TES gene at 7q31.1 is methylated in tumours and encodes a novel growth-suppressing LIM domain protein. Oncogene 20: 2844-2853, 2001.

10. Mueller W, Nutt CL, Ehrich M, Riemenschneider MJ, von Deimling A, van den Boom D and Louis DN: Down-regulation of RUNX3 and TES by hypermethylation in glioblastoma. Oncogene 26: 583-593, 2007.

11. Louis DN, Ohgaki H, Wiestler OD, Cavenee WK, Burger PC, Jouvet A, Scheithauer BW and Kleihues P: The 2007 WHO classification of tumours of the central nervous system. Acta Neuropathol 114: 97-109, 2007.

12. Miller SA, Dykes DD and Polesky HF: A simple salting out procedure for extracting DNA from human nucleated cells. Nucleic Acids Res 16: 1215, 1988.

13. Ma HW, Weng D, Chen Y, Huang W, Pan K, Wang H, Sun J, Wang Q, Zhou Z, Wang $\mathrm{H}$ and Xia J: Extensive analysis of D7S486 in primary gastric cancer supports TESTIN as a candidate tumor suppressor gene. Mol Cancer 9: 190, 2010.

14. Majchrzak-Celińska A, Paluszczak J, Szalata M, Barciszewska AM, Nowak S, Kleszcz R, Sherba A and Baer-Dubowska W: The methylation of a panel of genes differentiates low-grade from high-grade gliomas. Tumour Biol 36: 3831-3841, 2015.

15. Martinez R, Martin-Subero JI, Rohde V, Kirsch M, Alaminos M, Fernandez AF, Ropero S, Schackert G and Esteller M: A microarray-based DNA methylation study of glioblastoma multiforme. Epigenetics 4: 255-264, 2009.

16. Gunduz E, Gunduz M, Beder L, Nagatsuka H, Fukushima K, Sutcu R, Delibas N, Yamanaka N, Shimizu K and Nagai N: Down-regulation of TESTIN and its association with cancer history and a tendency toward poor survival in head and neck squamous cell carcinoma. Arch Otolaryngol Head Neck Surg 135: 254-260, 2009.

17. Weeks RJ, Kees UR, Song S and Morison IM: Silencing of TESTIN by dense biallelic promoter methylation is the most common molecular event in childhood acute lymphoblastic leukaemia. Mol Cancer 9: 163, 2010.

18. Bai Y, Zhang QG and Wang XH: Down-regulation of TES by hypermethylation in glioblastoma reduces cell apoptosis and predicts poor clinical outcome. Eur J Med Res 19: 66, 2014. 